\title{
A scheme of estimating mobile traffic data without coarse-grained process using conditional SR-GAN
}

\author{
Tomoki Tokunaga ${ }^{1, \text { a) }}$ and Kimihiro Mizutani ${ }^{1}$ \\ ${ }^{1}$ Graduate School of Science and Engineering, Kindai University \\ 3-4-1 Kowakae, Higashi Osaka, Osaka 577-0818, Japan \\ a)2033340424m@kindai.ac.jp
}

\begin{abstract}
In recent years, a scheme for generating a large amount of mobile traffic data has been proposed. In the state-of-the-art of the schemes, Generative Adversarial Networks (GANs) is used to transform a large amount of traffic data into a coarse-grained representation and to generate the original traffic data from the coarse-grained data. However, in order to generate the original traffic data, the coarse-grained data must be preserved and it takes waste storage cost. In this paper, we propose a scheme for generating the mobile traffic data without requiring a coarse-grained process by using Conditional SR-GAN. In evaluation using real mobile traffic data, our proposed scheme not only does reduce the storage cost by more than $25 \%$ compared to the traditional scheme, but also can generate the original mobile traffic data with $94 \%$ accuracy.
\end{abstract}

Keywords: conditional GAN, SR-GAN, traffic data management Classification: Network Management/Operation

\section{References}

[1] Erricson surveys, https://www.ericsson.com/en/mobility-report/reports/ june-2019/mobile-data-traffic-outlook

[2] C. Zhang, X. Ouyang, and P. Patras, "ZipNet-GAN: inferring fine-grained mobile traffic patterns via a generative adversarial neural network," Proc. ACM CoNEXT, pp. 363-375, 2017. DOI: 10.1145/3143361.3143393

[3] I. Goodfellow, J. Pouget-Abadie, M. Mirza, B. Xu, D. Warde-Farley, S. Ozair, A. Courville, and Y. Bengio, "Generative adversarial nets," Proc. 27th International Conference on Neural Information Processing Systems (NIPS), pp. 2672-2680, 2014.

[4] M. Mehdi and S. Osinderoet, "Conditional generative adversarial nets," arXiv preprint arXiv:1411.1784, 2014.

[5] C. Ledig, L. Theis, F. Huszar, J. Caballero, A. Cunningham, A. Acosta, A. Aitken, A. Tejani, J. Totz, Z. Wang, and W. Shi, "Photo-realistic single image super-resolution using a generative adversarial network," Proc. IEEE Conference on Computer Vision and Pattern Recognition (CVPR), pp. 4681-4690, 2017. DOI: $10.1109 /$ cvpr.2017.19 
[6] K. He, X. Zhang, S. Ren, and J. Sun, "Deep residual learning for image recognition," Proc. IEEE Conference on Computer Vision and Pattern Recognition, pp. 770-778, 2016. DOI: 10.1109/cvpr.2016.90

[7] K. Simonyan and A. Zisserman, "Very deep convolutional networks for largescale image recognition," arXiv preprint arXiv:1409.1556, 2014.

[8] https://pytorch.org

[9] G. Barlacchi, M. De Nadai, R. Larcher, A. Casella, C. Chitic, G. Torrisi, F. Antonelli, A. Vespignani, A. Pentland, and B. Lepri, "A multi-source dataset of urban life in the city of Milan and the province of Trentino," Scientific Data, Nature, 2015. DOI: 10.1038/sdata.2015.55

\section{Introduction}

Mobile traffic data has increased rapidly in recent years due to the dramatic spread of mobile devices and online services. According to a survey by Ericsson, the amount of monthly mobile traffic data is expected to reach 131 exabytes by 2024 [1]. In order to handle the large amount of future mobile traffic data, it is necessary to analyze the mobile traffic data and to increase the number of mobile base stations. However, since the amount of mobile traffic data increases enormously with the size and population density of the target area, its high storage cost becomes a problem. In order to solve this problem, a scheme for generate a large amount of traffic data has been proposed. Among them, many schemes based on deep neural network have been proposed due to recent advances in its technique.

In particular, the state-of-the-art of the schemes, ZipNet-GAN [2] transforms a large amount of traffic data into a coarse-grained representation and generate the original traffic data from the coarse-grained data. In ZipNet-GAN, a mobile traffic data can be represented in the three dimensional form ( $x, y$, volume) where $x$ and $y$ are longitude and latitude, and volume is the amount of traffic data at that point. If we preserve the time series of mobile traffic data covered by a rectangular area (e.g., a grid cell or traffic map), the storage cost can be expressed as $X \cdot Y \cdot T$ where $X, Y$, and $T$ are the ranges of longitude, latitude and time. In a coarse-grained representation, the mobile traffic data $X \cdot Y \cdot T$ is transformed to a coarse-grained data $(X / C) \cdot(Y / C) \cdot T$ where $C$ is a constant value. Noting that the original paper proposed ZipNet-GAN used $C=10$. From the coarse-grained data $(X / C) \cdot(Y / C) \cdot T$, ZipNet-GAN generates the original traffic data $X \cdot Y \cdot T$. As a result, the original traffic data can be generated accurately, but the storage cost for coarse-grained data increases as the number of rectangular traffic data area and the time span increase.

To solve the storage cost problem of coarse-grained data, we propose accurate mobile traffic generation scheme without coarse-grained data using Conditional SRGAN. Our proposed scheme generating the original traffic data from the random numbers and the target time data (label). With this scheme, the storage cost of the mobile traffic data $X \cdot Y \cdot T$ can be reduced to only $T$ time labels noting that the random numbers are temporary used and do not need to be preserved. In other words, our scheme need not preserve a coarse-grained data, so that it can generate mobile traffic data more efficiently than ZipNet-GAN. 


\section{Proposed scheme Conditional SR-GAN}

\subsection{Conditional SR-GAN}

In this paper, we propose a scheme using Conditional SR-GAN for generating mobile traffic data without coarse-grained data. GANs [3] is a latest unsupervised learning framework for generating artificial data from random numbers. In general, GANs consists of two neural network models, generator $G$ generating target artificial data from random numbers, and discriminator $D$ estimating the probability that the output of $G$ is derived from real training data rather than the artificial data.

Fig. 1 shows an abstraction of the Conditional SR-GAN for generating mobile traffic data in our proposed scheme. The Conditional Super-Resolution GAN is composed of a combination of Conditional GAN (C-GAN) [4] and Super-Resolution GAN (SR-GAN) [5]. The Conditional GAN is a scheme of generating a original data from a certain label. The SR-GAN has a same feature of ZipNet-GAN, but it outperforms ZipNet-GAN in term of generating accurate original data. The Conditional SR-GAN generates mobile traffic data from normal random numbers and the target time data (label), directly. Therefore, the storage cost takes only the sizes of list of target time data (label). The formula for the Conditional SR-GAN is shown following:

$$
\begin{aligned}
\min _{G} \max _{D} V(G, D)= & E_{x \sim \operatorname{Pr}(x)}[\log D(x \mid L)] \\
& +E_{z \sim P_{n}(z)}[\log (1-D(G(z \mid L)))]
\end{aligned}
$$

In Conditional SR-GAN, the generator $G$ takes random data seed (e.g., Gaussian or uniformly distributed) $z \sim P n(z)$ with a target time label $L$, and produces an output $\hat{x}$ that aims to follow a target mobile traffic data. On the other hand, the discriminator $D$ randomly picks the data generated by $G$ (i.e., $\hat{x} \sim G(z \mid L)$ ), and others sampled from the target data distribution (i.e., $x \sim P_{r}(x)$ ) with its label $L$, and takes a judgement for them.

In detail, $G$ is trained to produce data whose distribution is as close as possible to $P_{r}(x)$ for maximising the probability that $D$ makes mistakes. On the other hands, $D$ is trained to maximise the probabilities of correctly judging whether $\hat{x}$ is fake or $x$ is real.

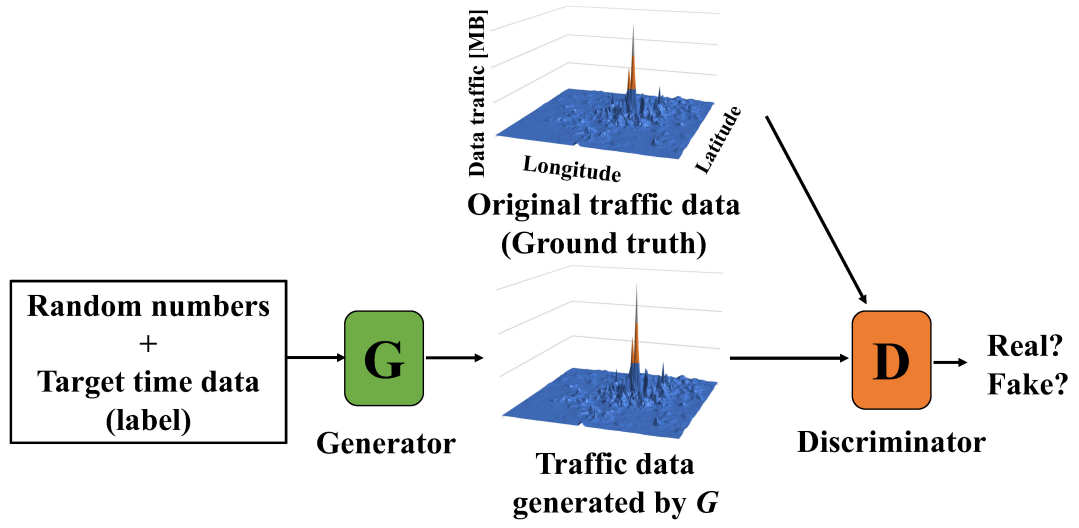

Fig. 1. The abstraction of a scheme of generating mobile traffic data by using Conditional SR-GAN 
Once trained, the generator $G$ can produce a traffic data from random numbers (noisy inputs) $z$ with target label, and its output is closer to the target traffic data. Applying the label for GANs architecture enables the generator $G$ to output the original traffic data from only random numbers $z$ and target time label $L$.

\subsection{The concrete architecture of Conditional SR-GAN}

The generator of the Conditional SR-GAN consists of multiple ResNet [6] layers and pixel snuffer layers, which is based on SR-GAN's generator outputting [5]. It generates the traffic data from input data consisting of both normal random numbers and target time label (e.g., 2013.11.01.23.50). Next, the discriminator consists of only multiple ResNet layers such as VGG architecture [7]. It takes as input data a mixture of both the original traffic data and the traffic data generated by $G$, and it judges whether each input is original data or not. Therefore, the output of the discriminator is thus several $[0,1]$ values. In the learning phase of the Conditional SR-GAN, the discriminator tries to minimize the error in the judgements. In contrast, the generator not only tries to minimize the error between the output data and the original traffic data, but also maximizes the discriminator's output error. The combination of maximizing and minimizing errors during the generator's learning phase contributes to highly accurate generator's output. Using Conditional SR-GAN, the mobile traffic data can be generated from just the normal random numbers and the target time data (label). Therefore, the storage cost of traffic data reduce to just the amount of the time labels.

\section{Evaluation}

We evaluated the proposed scheme with real mobile-traffic data. We implemented our scheme using Python3 and the PyTorch libraries [8]. The input data for the generator consists of two elements: the time label of the target traffic data and a random number. We changed both elements to carry out an exhaustive verification of our scheme. We changed the number of random numbers from 10 to 100 to 1,000. Next, We used the four types of representations of the time labels: decimal time, binary time, ordered decimal time, and ordered binary time. Decimal time corresponds to the simple time representation consisting of year, month, day, hour, and minute (e.g., 201311012350). Binary time is just the binarized representation of the decimal time. Ordered decimal time indicates the number of data from the first data in all data sets. For example, when the range of the traffic data set is [201311010000, 201401012350], the ordered decimal time corresponding to 20131101000 is 1 . Ordered binary time is the binarized form of ordered decimal time. The data set we used for this evaluation consisted of real mobile-traffic data collected from Telecom Italia's Big Data Challenge [9]. This includes two months of traffic data in Toronto sampled every 10 minutes between 1 November 2013 and 1 January 2014. The coverage area of the mobile traffic data is divided into $117 \times$ 98 squares, with each point on the map indicating the traffic volume. Note that 117 $\times 98$ is transformed to $100 * 100$ in Conditional SR-GAN.

Finally, we use MSE (Mean Square Error) for generator loss to minimize the 
settings, we trained the Conditional SR-GAN to generate correct $100 \times 100$ traffic volume map from random numbers and the corresponding time labels. The number of training iterations (epoch) is 400, and for parameters such as the learning rate, we used the values recommended from the PyTorch libraries. This evaluation rival for comparing performances is SR-GAN, which is fully upward compatible with ZipNet-GAN. Noting that SR-GAN's generator generates the original traffic data from coarse-grained data of $10 \times 10$.

Fig. 2 shows the MSE between the generator's output in the proposed scheme and the original traffic data against the number of epochs. The result show that as the number of random numbers decreases, the convergence speed is improved (left of Fig. 2). When the number of random numbers is fixed at 10 , the convergence speeds were different for the different types of target time label representations (right of Fig. 2). Ordered decimal time was considered to be unstable due to the small number of elements in the input vectors, and from this fact, we concluded that the binary notation label is more suitable for label representation because it increases the number of elements in the input vectors. We also compared the output data of SR-GAN with the coarse-grained data $10 \times 10$ as input (right of Fig. 2). It can be seen that there is no difference in the value of MSE convergence between our proposed scheme (Conditional SR-GAN) and ZipNet-GAN (i.e., SR-GAN).

Fig. 3 is a snapshot of the output data of our scheme with the number of random numbers as 10 and binary times as input for November 1 in 2013 (left of Fig. 3) and is a snapshot of the original traffic data for same time (right of Fig. 3). We observed that our proposed scheme delivers remarkably accurate generates, as the texture and
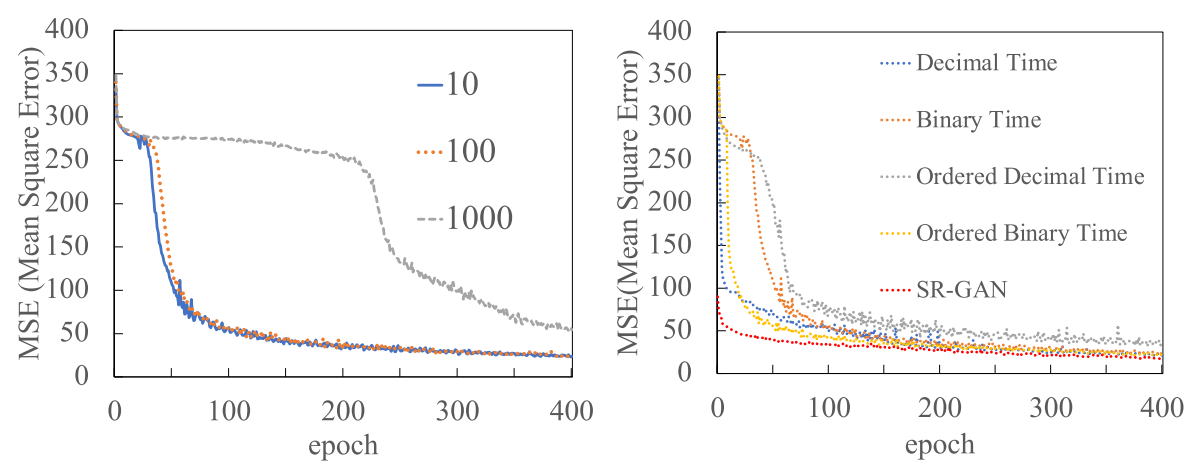

Fig. 2. MSE between the generator output in our proposed scheme and the original traffic data against the number of epochs.
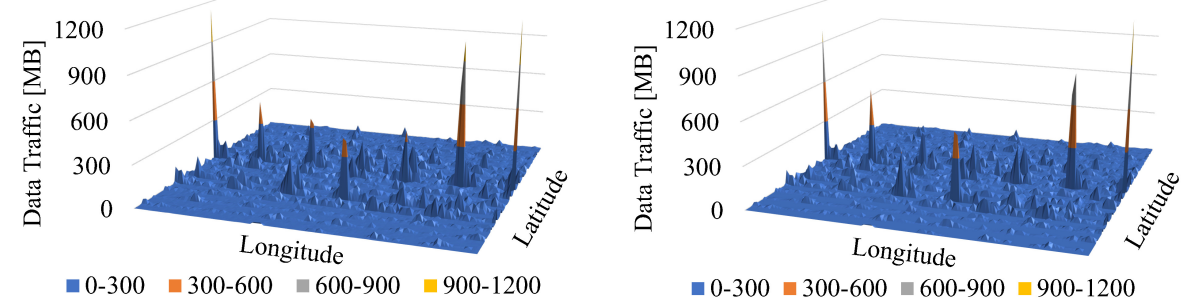

Fig. 3. A snapshot of estimated/original traffic data for Tronto's mobile traffic data in November 1 in 2013. 
details are almost perfectly recovered. In detail, our proposed scheme achieves $94 \%$ accuracy for original mobile traffic data.

Finally, we compared the storage costs for ZIP compression, SR-GAN, and our proposed scheme. The size of the original mobile-traffic data was 1GB. Applying ZIP compression for compressing original traffic data, the volume was reduced to 473 MB. Next, applying SR-GAN, the traffic volume after the coarse-grained process was $10 \mathrm{MB}$. However, storage is also required for the generator model:29 MB noting that SR-GAN must preserve its generator's model for generating coarse-grained data to original data. Therefore, the storage size for SR-GAN was $39 \mathrm{MB}$ noting that our scheme needs storage only for the generator model and the time labels for generating the original traffic data. Then, the model object is the same size as for SR-GAN and the amount of the time labels is $2 \mathrm{~KB}$, therefore, they take only $29.2 \mathrm{MB}$. The results show that our scheme can reduce the storage cost the mobile traffic data by more than $25 \%$ compared to SR-GAN. We also evaluated with the Milan data set. In Milan data set, The size of the original mobile-traffic data was $813 \mathrm{MB}$. The storage size for SR-GAN took 37.1 MB while our scheme takes storage size was 29.2 MB. The result indicates that our scheme can reduce the storage cost the mobile traffic data by more than $20 \%$ compared to SR-GAN. Summarizing both data set compression results, our scheme could reduce more than $20 \%$ on two data sets.

\section{Conclusion}

Mobile traffic data has increased rapidly in recent years due to the dramatic spread of mobile devices and online services. And its storage cost becomes much higher. ZipNet-GAN, traditional scheme, solves the storage cost problem by generating traffic data from coarse-grained data, but the coarse-grained data must be preserved. We proposed here a scheme for generating mobile traffic data without requiring coarsegrained data. Our proposed scheme consists of Generative Adversarial Networks (GANs) architecture that combines C-GAN and SR-GAN. The proposed Conditional SR-GAN is can generate the original traffic data correctly, and the storage cost is relatively modest. In evaluation, we concluded that our proposed scheme not only does reduce the storage cost for real mobile traffic data by more than $20 \%$ compared to ZipNet-GAN, but also does provide an accurate traffic data generation of the original mobile traffic data. For the future, we will improve the accuracy by customizing GAN and calculation efficiency with distributed learning manner.

\section{Acknowledgments}

This work was supported by JSPS KAKENHI Grant Number JP20K19791. 